\title{
The dynamic interactions between chemotherapy drugs and plasmid DNA investigated by atomic force microscopy
}

\author{
Mi $\mathrm{Li}^{1}$, Lianqing $\mathrm{Liu}^{1 *}$, Xiubin $\mathrm{Xiao}^{2}$, Ning $\mathrm{Xi}^{1,3^{*}}$ and Yuechao Wang ${ }^{1}$
}

\begin{abstract}
The advent of atomic force microscopy (AFM) provides a powerful tool for imaging individual DNA molecules. Chemotherapy drugs are often related to DNAs. Though many specific drug-DNA interactions have been observed by AFM, knowledge about the dynamic interactions between chemotherapy drugs and plasmid DNAs is still scarce. In this work, AFM was applied to investigate the nanoscale interactions between plasmid DNAs and two commercial chemotherapy drugs (methotrexate and cisplatin). Plasmid DNAs were immobilized on mica which was coated by silanes in advance. AFM imaging distinctly revealed the dynamic changes of single plasmid DNAs after the stimulation of methotrexate and cisplatin. Geometric features of plasmid DNAs were extracted from AFM images and the statistical results showed that the geometric features of plasmid DNAs changed significantly after the stimulation of drugs. This research provides a novel idea to study the actions of chemotherapy drugs against plasmid DNAs at the single-molecule level.
\end{abstract}

Keywords: atomic force microscopy, plasmid DNA, drug, methotrexate, cisplatin

\section{INTRODUCTION}

Cancer is a leading cause of death in both more and less economically developed countries [1]. We know that cancer treatment is a three-legged stool sitting on a base of surgery, radiation therapy, and chemotherapy [2]. However, chemotherapy is the main treatment of cancers [3]. Many chemotherapy drugs target DNAs or influence DNAs [4]. Hence, detailed information about drug-DNA interactions is critical to our understanding of drug actions and can help us to design better drugs.
However, traditional biochemical experiments which are done in test tube with bulk molecules are unable to observe the behaviors of single biological molecules after the stimulation of drugs [5]. The advent of atomic force microscopy (AFM) provides a powerful tool for directly imaging single biological molecules in their native states with nanometer spatial resolution, such as DNAs [6], antibodies [7], and membrane proteins [8]. With AFM, we can directly observe the morphological changes of single DNAs after the treatment with drugs in aqueous environment [9]. Besides morphology imaging, AFM can also measure the mechanics of single DNA by stretching the molecule $[10,11]$. Especially the development of high-speed AFM brings new opportunities for studying the structure and dynamics of single biomolecules in action [12]. In the past decade, the applications of AFM in DNA-related research have provided plenty of novel insights into the behaviors of single DNAs [13-18]. Researchers have applied AFM to investigate the interactions between diverse drugs and DNA molecules [19-24], but these studies are commonly done with linear DNAs. Information about the dynamic interactions between chemotherapy drugs and plasmid DNAs at the nanoscale is still scarce. Besides, there is still a lack of simple methods for extracting the quantitative information of the plasmid DNA from AFM images. Methotrexate [25] and cisplatin [19] are two clinically-used chemotherapy drugs which interact with DNAs. To our knowledge, so far how methotrexate and cisplatin cause the dynamic changes of single plasmid DNAs is still unknown. In this work, with the use of AFM, the dynamic changes in morphology and geometry of individual plasmid DNAs

\footnotetext{
${ }^{1}$ State Key Laboratory of Robotics, Shenyang Institute of Automation, Chinese Academy of Sciences, Shenyang 110016, China

${ }^{2}$ Department of Lymphoma, Affiliated Hospital of Military Medical Academy of Sciences, Beijing 100071, China

${ }^{3}$ Department of Electrical and Computer Engineering, Michigan State University, East Lansing, MI 48824, USA

*Corresponding authors (emails: lqliu@sia.cn (Liu L); xin@egr.msu.edu (Xi N))
} 
after the treatment with methotrexate and cisplatin were revealed.

\section{MATERIALS AND METHODS}

\section{Mica modification}

Mica was coated by aminopropyltriethoxy silane (APTES) [6] via the method of vapor deposition. APTES was purchased from Sigma-Aldrich (Saint Louis, MO, USA). The detailed procedure for mica modification was as follows. A piece of mica $(1 \mathrm{~cm} \times 1 \mathrm{~cm})$ was attached to a metal disk via a double faced adhesive and then the top layer of the mica was removed. The metal disk was placed in a Petri dish and then put into a glass desiccator. Twenty microliter of APTES was dropped onto another fresh Petri dish and then put into the glass desiccator. The glass desiccator was lidded to incubate for $12 \mathrm{~h}$. After the incubation, the mica was washed by ultrapure water $(18.2 \mathrm{M} \Omega \mathrm{cm}$ ) (Direct-Q, Merck Millipore, USA) and then dried by compressed air. The treated mica was used for immobilizing plasmid DNAs.

\section{Sample preparation}

Methotrexate $\left(25 \mathrm{mg} \mathrm{mL}^{-1}\right)$ and cisplatin $\left(1 \mathrm{mg} \mathrm{mL}^{-1}\right)$ were obtained from the Affiliated Hospital of Military Medical Academy of Sciences (Beijing, China). ChR plasmid DNA (878 $\mathrm{ng} \mathrm{LL}^{-1}$ ) was purchased from Takara company (Dalian,
China). The detailed procedure for sample preparation was as follows. A drop $(1 \mu \mathrm{L})$ of plasmid DNA solution was diluted with $1 \mathrm{~mL}$ of ultrapure water to a molar concentration of $0.3 \mu \mathrm{mol} \mathrm{L}^{-1}$. The chemotherapy drugs were diluted with ultrapure water to concentrations of 1 and $5 \mathrm{ng} \mathrm{L}^{-1}$, respectively. The molar concentrations of methotrexate were 2.2 $\left(1 \mathrm{ng} \mu \mathrm{L}^{-1}\right)$ and $11 \mu \mathrm{mol} \mathrm{L}^{-1}\left(5 \mathrm{ng} \mu \mathrm{L}^{-1}\right)$. The molar concentrations of cisplatin were $3.3\left(1 \mathrm{ng} \mu \mathrm{L}^{-1}\right)$ and $16.5 \mu \mathrm{mol} \mathrm{L}^{-1}$ (5 $\mathrm{ng} \mu \mathrm{L}^{-1}$ ). The drug solution and plasmid DNA solution were mixed and then incubated for a certain time $(30 \mathrm{~min}$, $60 \mathrm{~min}$ ) at room temperature. The plasmid DNA solution (without or with drug) was dropped on the APTES-coated mica and incubated for $1 \mathrm{~min}$. The mica was washed with ultrapure water for three times and dried in compressed air. The sample was put on the AFM stage for imaging.

\section{AFM imaging}

A Multimode AFM (Bruker, Santa Barbara, CA, USA) was used in the experiments, as shown in Fig. 1a. There was an optical microscope at the top of AFM to observe the AFM probe and the sample. AFM images were obtained at a tapping mode in air. The type of probe used was TESP (Bruker, Santa Barbara, CA, USA) with a nominal spring constant of $42 \mathrm{~N} \mathrm{~m}^{-1}$ (Fig. 1b). The driving frequency was about $326.5 \mathrm{kHz}$ (Fig. 1c). The scan rate was $1 \mathrm{~Hz}$. The
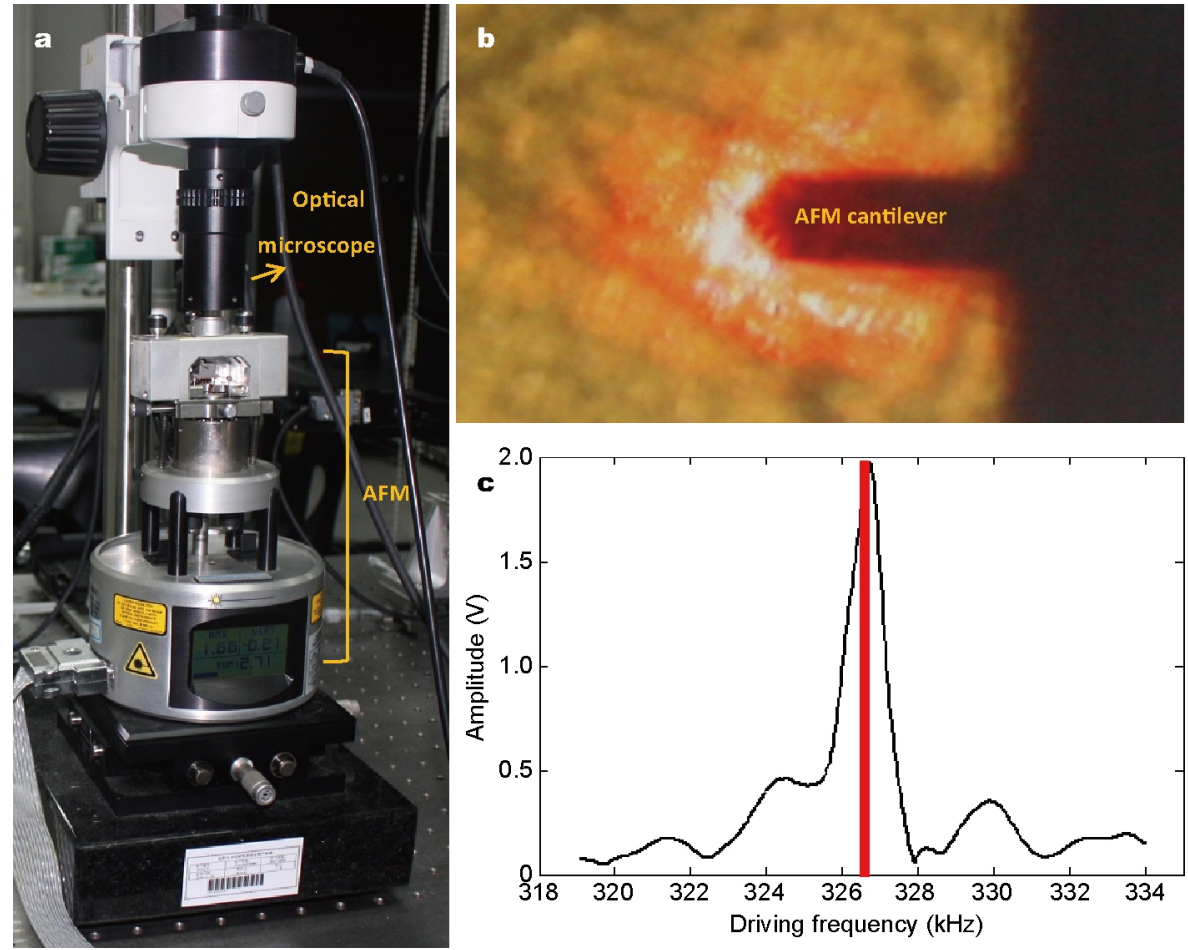

Figure 1 The experimental platform. (a) An actual photograph of AFM. (b) Optical image of the AFM cantilever. (c) Tuning the driving frequency of AFM cantilever. 
scan line was 256 and the sampling point for each scan line was 256. In order to obtain the scan force of the tapping cantilever, the force curves were firstly obtained on a rigid substrate and the deflection sensitivity $\left(\mathrm{nm} \mathrm{V}^{-1}\right)$ of the cantilever was calibrated from the force curves. The deflection of cantilever during tapping mode was computed from the deflection sensitivity and the amplitude (Fig. 1c). Then the scan force was obtained according to the Hooke's law ( $F=k x$, where $k$ is the spring constant of the cantilever and $x$ is the deflection of the cantilever). By modulating the amplitudes, different scan forces were obtained. About 50 AFM images of the plasmid DNAs were obtained from the control group (without the stimulation of drugs). For each situation of the drug group (with different drug concentrations and incubation times), also about 50 AFM images were obtained. These AFM images were then used to analyze the dynamic changes of the geometric features of plasmid DNAs.

\section{RESULTS AND DISCUSSION}

Fig. 2 shows the images of the plasmid DNAs on silanized mica by AFM. Firstly, we investigated the images of plasmid DNAs on bare mica which was not treated by APTES, as shown in Fig. 2a, b. Fig. 2a is the AFM image of bare mica and we can see that its surface is very clean. Fig. 2b shows the AFM image of the plasmid DNAs on bare mica. The red curve in Fig. $2 \mathrm{f}$ is obtained from the section curve along the red line in Fig. 2b, showing that the height of the DNA is $\sim 2 \mathrm{~nm}$ which is significantly larger than that of a single DNA measured by AFM. The theoretical diameter of the double helix DNA is $2 \mathrm{~nm}$ [26]. But in AFM imaging, due to the compression of DNA caused by the AFM tapping tip, the height of a single DNA $(0.5-1 \mathrm{~nm}[9,27])$ is often much less than $2 \mathrm{~nm}$. In fact, the blue curve in Fig. $2 \mathrm{f}$ is obtained from the section curve of a single DNA along the blue line in Fig. 2e, showing that the height of the plasmid DNA is $0.4-0.6 \mathrm{~nm}$, significantly less than the result of Fig. 2b. Hence the result in Fig. $2 b$ is deemed to originate from multiple plasmid DNAs, indicating that the plasmid DNAs aggregated on bare mica. Because mica and DNA are both negatively charged [6], DNA will not tightly adhere to bare mica. After dropping DNA solution onto the bare mica, the DNAs on the mica can then aggregate during the subsequent drying process. Currently, there are two main methods for modifying mica to adsorb DNA, including divalent cation-assisted adsorption and APTES-based chemical functionalization $[28,29]$. Here, we used APTES to modify mica to yield a positively charged surface [6].
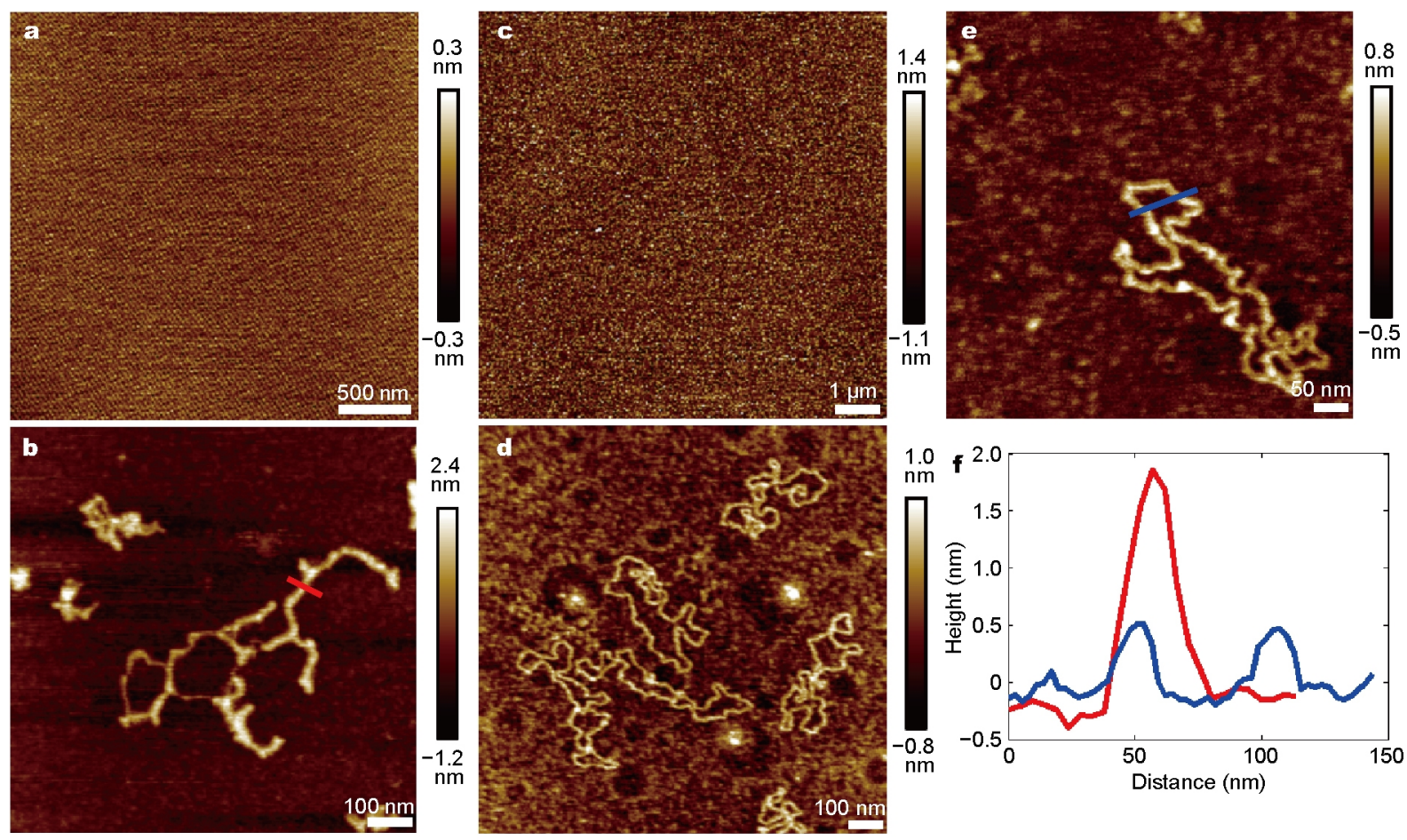

Figure 2 AFM imaging of plasmid DNA on mica. (a, b) AFM height images of bare mica (a) and plasmid DNAs on bare mica (b). (c) AFM height image of APTES-coated mica without DNA. (d, e) AFM height images of plasmid DNAs on APTES-coated mica (d, large size scan; e, small size scan of a single plasmid DNA). (f) Section curves taken along the red line in (b) and blue line in (e), respectively. 
Fig. 2c shows the AFM image of APTES-coated mica surface, which is also clean and smooth. Fig. $2 \mathrm{~d}$ shows the large size scan of plasmid DNAs on APTES-coated mica, clearly showing several plasmid DNAs on mica. We can see that the plasmid DNAs do not aggregate on the APTEScoated mica. Fig. 2e is the small size scan of a single plasmid DNA, clearly showing the circular structure of the plasmid DNA.

Fig. 3 shows the AFM images of single plasmid DNAs obtained at four different scan forces, 100, 200, 350 and 500 pN. Fig. 3a-c show the AFM images of a plasmid DNA obtained at three scan forces $(200,350$ and $500 \mathrm{pN})$ respectively. In order to analyze the influence of the scan force on the height of plasmid DNA measured by AFM, section curves at the same position of the DNA were obtained. The section curves taken along the red lines in Fig. $3 \mathrm{a}-\mathrm{c}$ are shown under each image. The section curves show the heights of two points on the plasmid DNA. When the scan force is $200 \mathrm{pN}$, the heights of the two points on the DNA are 0.6 and $1.1 \mathrm{~nm}$, respectively. When the scan force increases to $350 \mathrm{pN}$, the heights decrease to 0.55 and $0.9 \mathrm{~nm}$, respectively. When the scan force is $500 \mathrm{pN}$, the heights become 0.45 and $0.9 \mathrm{~nm}$. We can see that the increase of scan force caused the decreased height of DNA measured by AFM. The increase of scan force results in the larger compressed deformability of DNA, and thus the height of DNA decreases. Due to the fact that the large scan force may also cause damage to the DNA [14], in order to further examine the adequate scan force of DNA imaging, we then imaged plasmid DNAs under the scan force of $100 \mathrm{pN}$, as shown in Fig. 3d, e. We can see that single plasmid DNAs are clearly visible. The section curves taken along the red
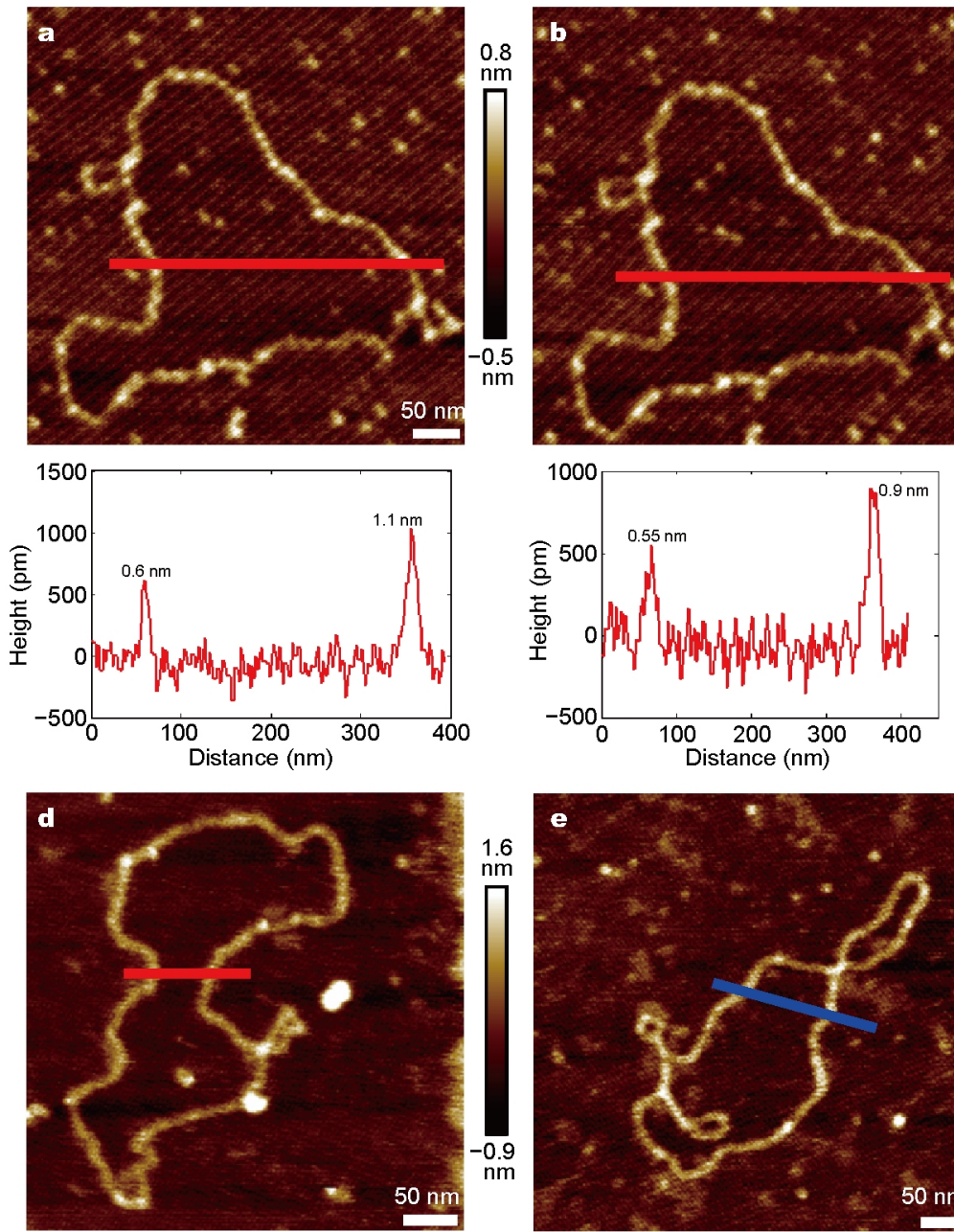
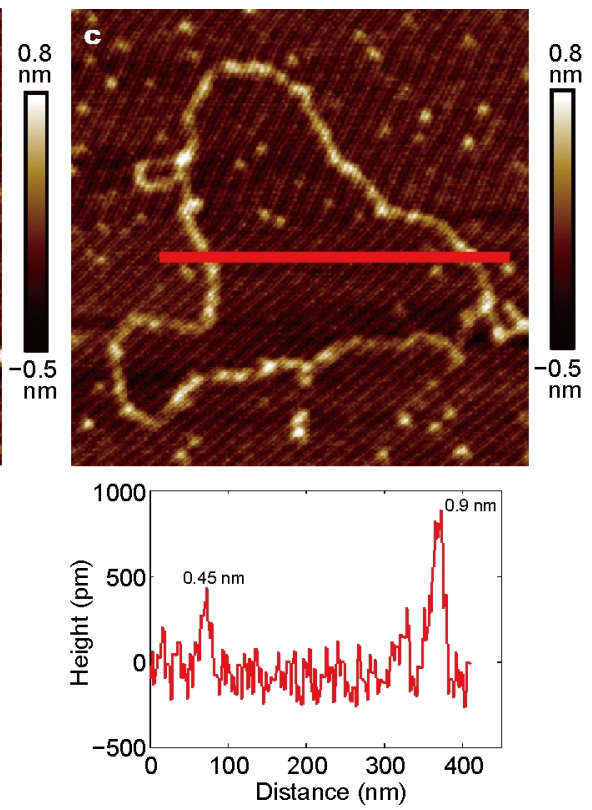

f

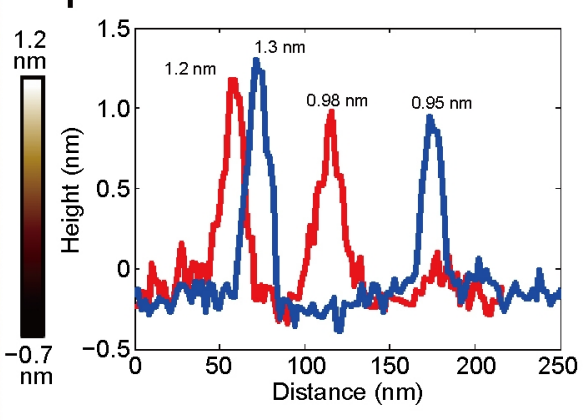

Figure 3 AFM height images of single plasmid DNAs obtained at different scan forces: (a) $200 \mathrm{pN}$, (b) $350 \mathrm{pN}$, (c) $500 \mathrm{pN}$ and their corresponding section curves taken along the red line in each image. (d, e) AFM height images under scan force of $100 \mathrm{pN}$. (f) Section curves taken along the red line in (d) and blue line in (e) respectively. 
line in Fig. $3 \mathrm{~d}$ and the blue line in Fig. 3e correspond to the red and blue curves in Fig. $3 \mathrm{f}$, respectively. We can see that the height of plasmid DNAs imaged under the scan force of $100 \mathrm{pN}$ is further larger than that under the scan force of $200 \mathrm{pN}$. In order to maximally avoid the mechanical damage to plasmid DNA from the scanning AFM tip, the scan force was set to $100 \mathrm{pN}$ in the following experiments.

Fig. 4 shows the AFM images of plasmid DNAs which were not treated by drugs, characterizing the geometric features of the plasmid DNAs. We can clearly see the circular single plasmid DNAs. Besides, there are a few cross points (denoted by the red arrows in Fig. 4) on the plasmid DNAs. Researchers have used persistence length [17], curvature [30] and bending angle [31] to represent the geometric feature of linear DNAs. Particularly, persistence length has been widely used to characterize the flexible mechanics of linear DNAs [32-34]. The persistence length of DNAs is commonly about $50 \mathrm{~nm}$ [32]. Several factors can cause the alterations of the DNA's persistence length, such as ionic strength [34], temperature [35] and drug binding [20]. In order to extract the persistence length of DNAs, we need to calculate the contour length and end-to-end distance of the DNA from AFM images [20]. For plasmid DNAs, because there are often many curls (Figs 5-7), it is difficult to calculate the contour length and thus measuring the persistence length of plasmid DNA is challenging. For plasmid DNAs, in 2013 Alonso-Sarduy et al. [9] used image processing to calculate the medium height of whole DNA. This method relies on converting the original AFM height image into a grayscale image according to a threshold and is relative complex. Here, we present a simple method to represent the geometric features of plasmid DNAs. Due to the circular characteristic of plasmid DNAs, we can draw a minimum rectangle which just contains the plasmid DNA, as shown in Fig. 4. Then the length and width of the rec- tangle can be used to present the geometric features of the DNA. This method has been used in characterizing single cells in AFM images [36]. Here we use it to represent the geometric information of single plasmid DNAs.

Fig. 5 shows the AFM images of plasmid DNAs after the stimulation of methotrexate. Two concentrations of methotrexate ( 1 and $5 \mathrm{ng} \mu \mathrm{L}^{-1}$ ) were used. Fig. $5 \mathrm{a}, \mathrm{b}$ are the images of plasmid DNAs treated by $1 \mathrm{ng} \mu \mathrm{L}^{-1}$ methotrexate for $30 \mathrm{~min}$. Comparing Fig. 5a, b with the AFM images of plasmid DNAs from the control group (Fig. 4), we can see that some plasmid DNAs after the stimulation have no significant morphological changes (Fig. 5a), while some plasmid DNAs have increased number of cross points (denoted by the red arrow in Fig. 5b). Then we increased the concentration of methotrexate. Fig. $5 \mathrm{c}-\mathrm{e}$ are the images of plasmid DNAs treated by $5 \mathrm{ng} \mu \mathrm{L}^{-1}$ methotrexate for $30 \mathrm{~min}$. At this situation, we can clearly see that multiple plasmid DNAs twine together (Fig. $5 \mathrm{c}$ ) and the number of cross points of the plasmid DNAs significantly increases (Fig. 5d, e). Further, we observed the dynamic dissociations of single supercoil plasmid DNAs after the stimulation of methotrexate, as shown in Fig. 6. Fig. 6a, b are the representative AFM images of supercoil plasmid DNAs without the treatment by methotrexate. The insets in Fig. $6 \mathrm{a}, \mathrm{b}$ are the section curves taken along the red lines. We can see that the height of DNAs is significantly larger than $2 \mathrm{~nm}$, indicating that they are bundled DNAs. A small plasmid DNA local loop is observed in Fig. 6a (denoted by the asterisk), which does not form supercoil structures. The height of the DNA local loop was about $0.6 \mathrm{~nm}$ (data not shown), which is comparable to the height of a single plasmid DNA. Besides, we can clearly see the helix structures of the supercoil plasmid DNAs (indicated by the arrows in Fig. 6a, b). These demonstrate that they are supercoil plasmid DNAs, showing that the plasmid DNAs can form
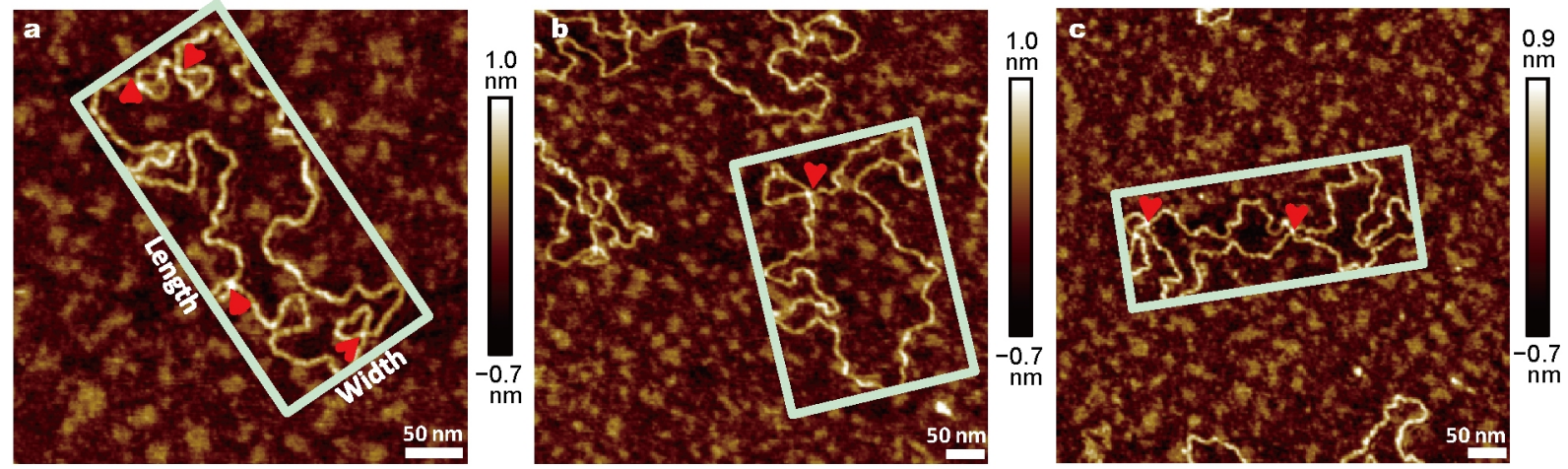

Figure 4 AFM height images of single plasmid DNAs from the control group (without drugs) and extraction of geometric information. The red arrows denote the cross points. 

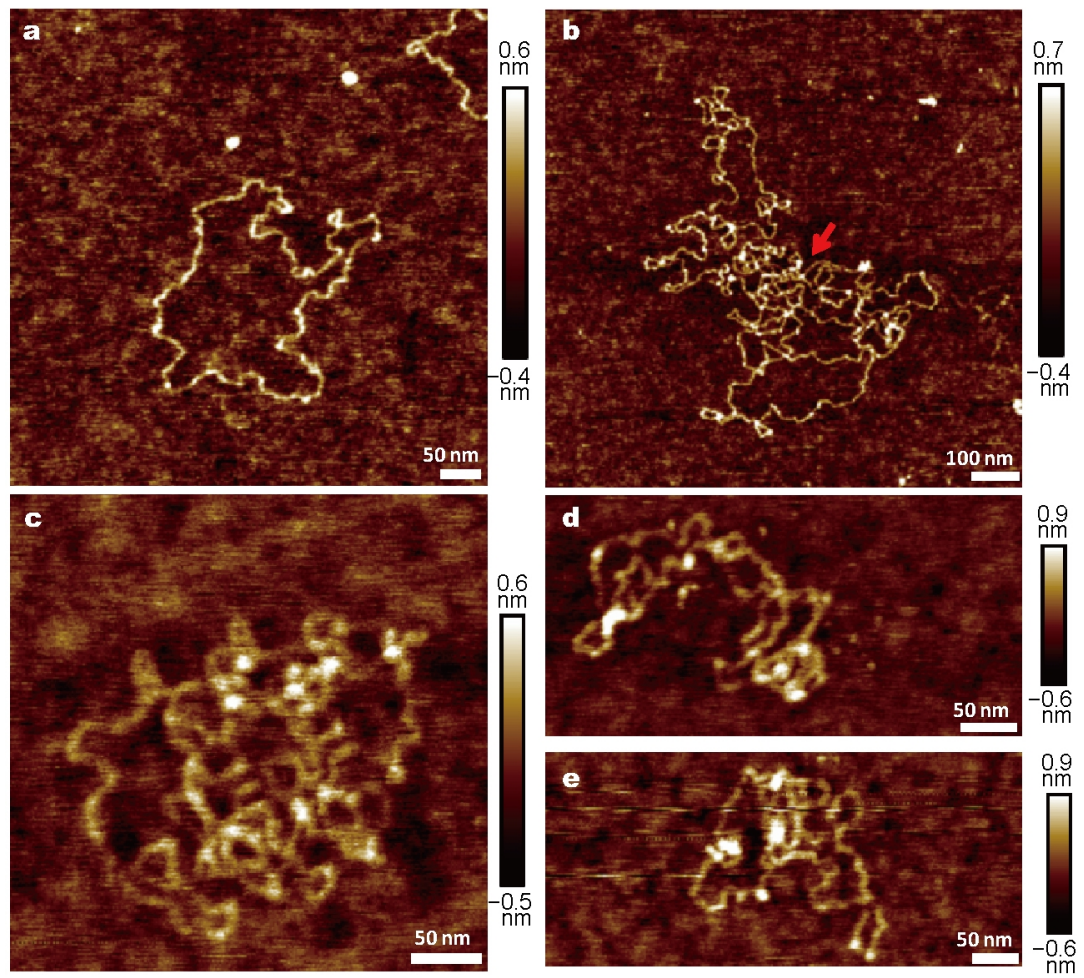

Figure 5 AFM height images of plasmid DNAs after the stimulation of methotrexate: (a, b) $1 \mathrm{ng} \mu \mathrm{L}^{-1}, 30 \mathrm{~min}$; (c-e) $5 \mathrm{ng} \mu \mathrm{L}^{-1}, 30 \mathrm{~min}$.
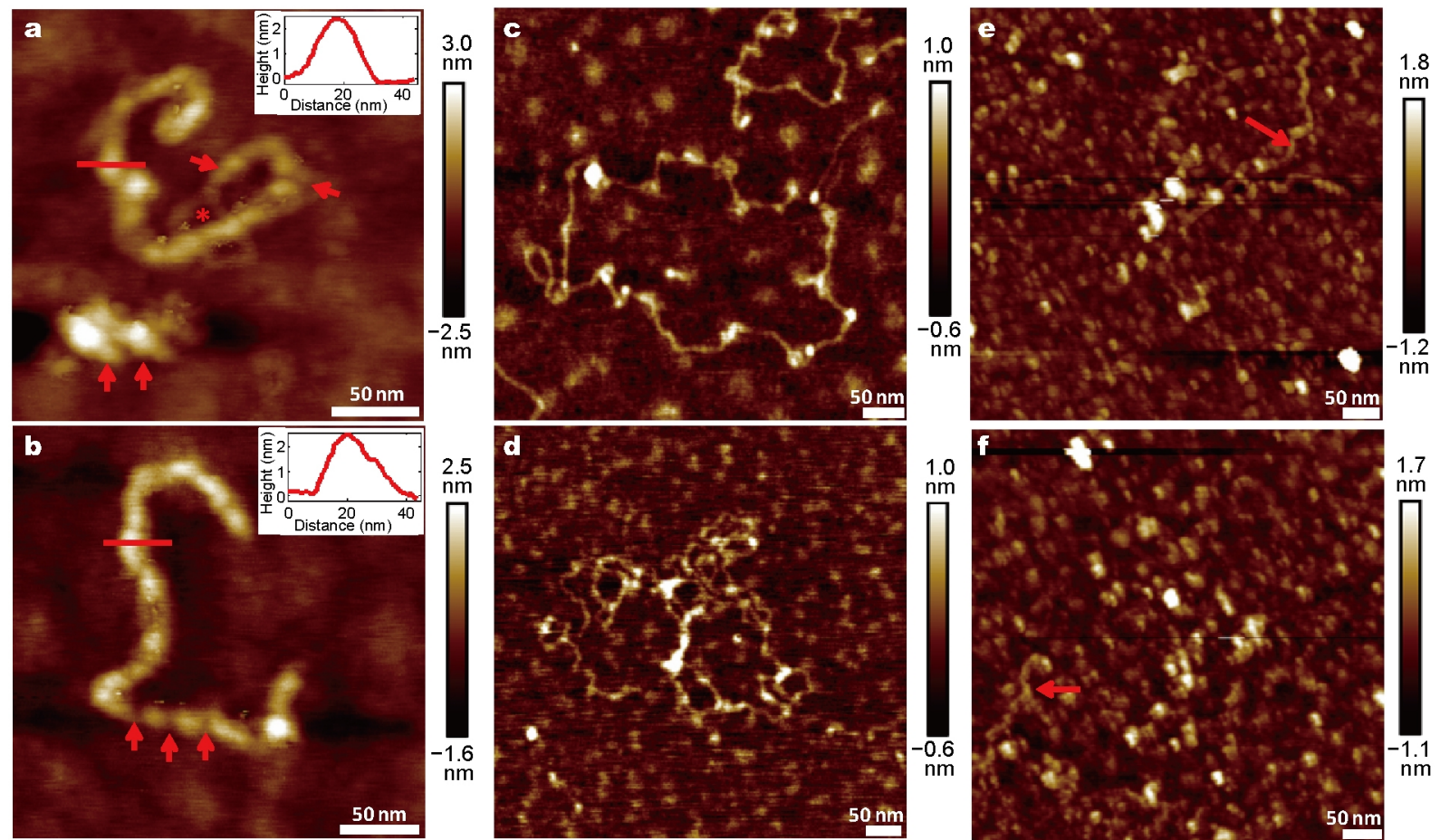

Figure 6 Dynamic changes of supercoil plasmid DNAs after the stimulation of methotrexate. (a, b) Control group without methotrexate. The arrows in (a, b) indicate the supercoil structures, and the insets in are the section curves taken along the red lines. (c, d) $5 \mathrm{ng} \mu \mathrm{L}^{-1}$ methotrexate, 30 min. (e, f) $5 \mathrm{ng} \mu \mathrm{L}^{-1}$ methotrexate, $60 \mathrm{~min}$. The arrows in (e, $\left.\mathrm{f}\right)$ indicate the linear DNAs. 
thick DNA rods by self-assembling. According to this characteristic, DNA molecules can be assembled into custom predesigned nanostructures and this is so-called origami $[37,38]$. Supercoiling and changes in the supercoiling state are ubiquitous in cellular behaviors and affect virtually all genomic processes [39]. Supercoiling can lead to either activation or repression of transcription [40]. Fig. 6c, d show the AFM images of the supercoil plasmid DNAs after treatment with $5 \mathrm{ng} \mu \mathrm{L}^{-1}$ methotrexate for $30 \mathrm{~min}$. We can see that after the treatment, supercoil plasmid DNAs disassembled and single plasmid DNAs are observed (Fig. 6c, d). Fig. $6 e$, $f$ show the AFM images of the supercoil plasmid DNAs after treatment with $5 \mathrm{ng} \mu \mathrm{L}^{-1}$ methotrexate for $60 \mathrm{~min}$. In this case, circular DNAs are not observed and disrupted linear DNAs are visible (denoted by the arrows). The results (Fig. 6) visually demonstrate that methotrexate can cause the disruption of supercoil plasmid DNAs. Methotrexate prevents cancer cells from sustaining purine and pyrimidine systems, which eventually inhibits the synthesis of DNA [25]. Studies have indicated that methotrexate is toxic to DNA [41]. Here, from Figs 5 and 6, we can clearly see that the stimulation of methotrexate can significantly cause damage to plasmid DNAs (especially the supercoil plasmid DNAs) at the single-molecule level, improving our understanding of the actions of methotrexate.
Fig. 7 shows the AFM images of plasmid DNAs after the stimulation of cisplatin. Fig. 7a-e are the AFM images of plasmid DNAs after treatment with $5 \mathrm{ng} \mu \mathrm{L}^{-1}$ cisplatin for $30 \mathrm{~min}$. We can see that the plasmid DNAs curl considerably and have increased number of cross points. Fig. $7 f-h$ are the AFM images of plasmid DNAs after treatment with $5 \mathrm{ng} \mu \mathrm{L}^{-1}$ cisplatin for $60 \mathrm{~min}$. At this situation, we can see that the plasmid DNAs are disrupted to linear DNAs. Fig. $7 \mathrm{i}-\mathrm{k}$ show the section curves taken along the red lines in Fig. $7 \mathrm{f}-\mathrm{h}$ respectively. We can see that the height of DNAs is comparable to that of single plasmid DNA measured by AFM (much less than $2 \mathrm{~nm}$ ), indicating that they are disrupted single plasmid DNAs. This is in contrary to Fig. 6a, b showing supercoil plasmid DNAs with the large height. Cisplatin has been used for treatment of numerous human cancers including bladder, head and neck, lung, ovarian, and testicular cancers [42]. It can crosslink with the purine bases on the DNA and cause DNA damage, which ultimately induce the apoptosis of cancer cells. In 2009, Hou et al. [19] investigated the effect of cisplatin on the morphology of linear DNAs by AFM imaging, showing that after the stimulation of cisplatin, DNA became more flexible and many local loops occurred. Here, we can also see that local micro-loops occur on the plasmid DNAs after the treatment with cisplatin (denoted by the arrows in Fig. 7a-e).
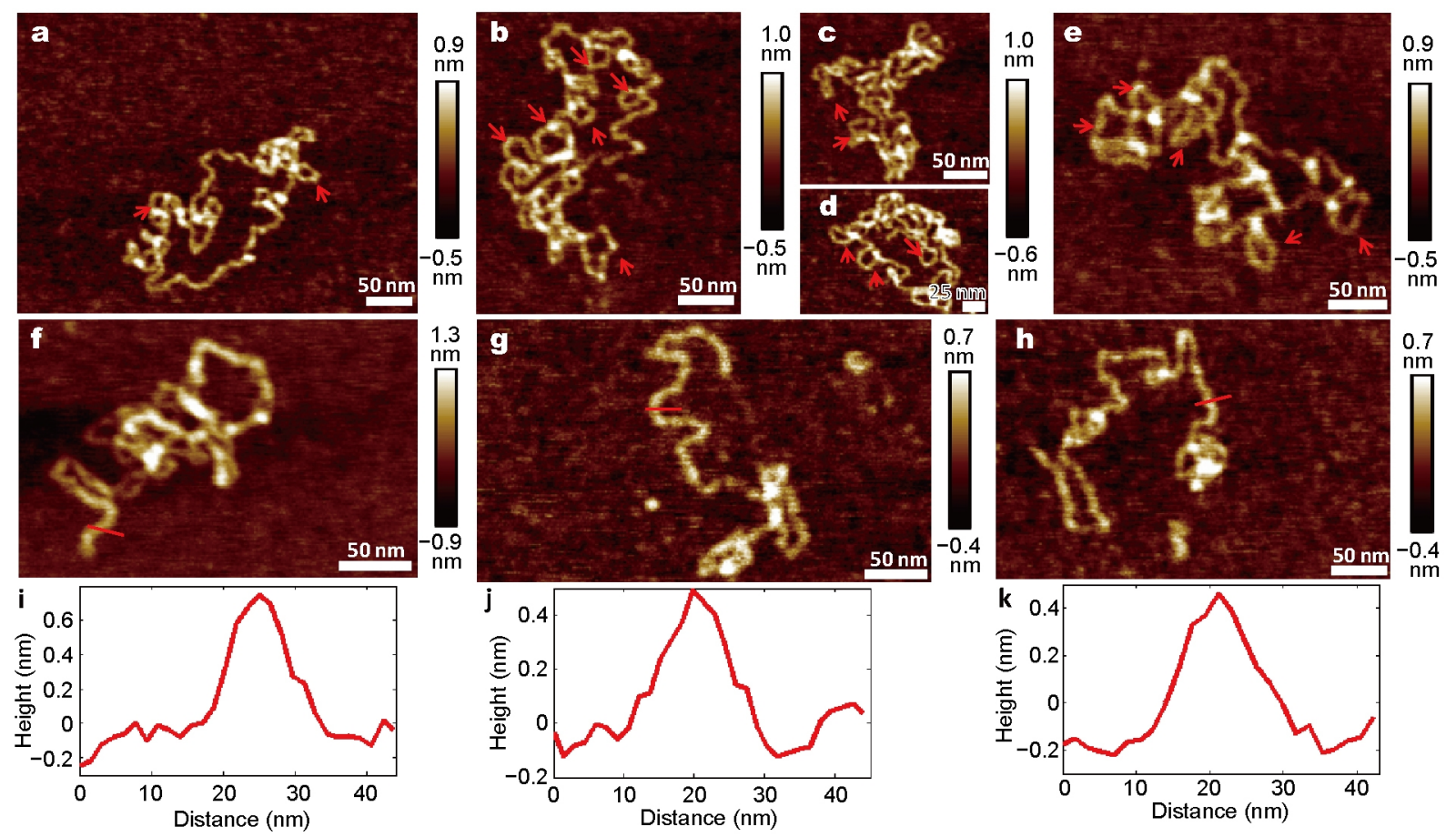

Figure 7 AFM height images of plasmid DNAs after the stimulation of cisplatin. (a-e) $5 \mathrm{ng} \mu \mathrm{L}^{-1}, 30 \mathrm{~min}$. (f-h) $5 \mathrm{ng} \mu \mathrm{L}^{-1}, 60 \mathrm{~min}$. (i-k) Section curves taken along the red lines in ( $\mathrm{f}-\mathrm{h})$, respectively. 

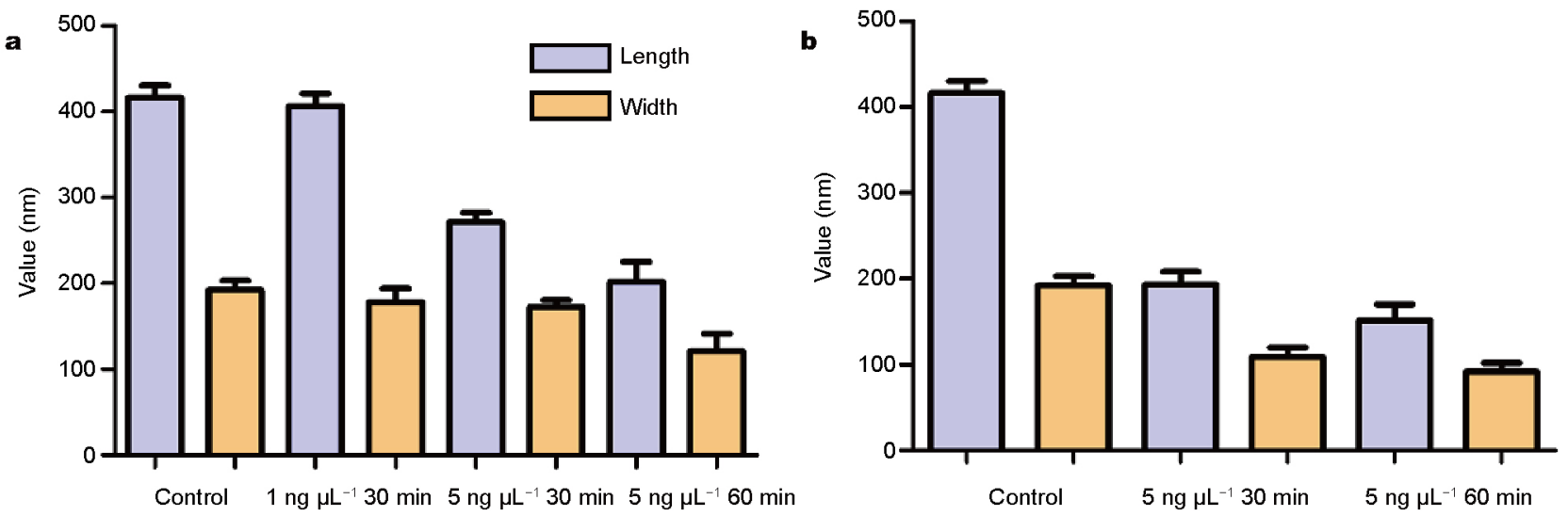

Figure 8 Changes of geometric features of plasmid DNAs after the stimulation of chemotherapy drugs (a, methotrexate; b, cisplatin) for different drug concentrations and incubation times. The values of length and width are shown as mean \pm SEM.

Though the plasmid DNAs from the control group also have local loops (denoted by the arrows in Fig. 4), but the number of local loops is significantly less than that treated by cisplatin, showing that cisplatin can cause the local curl of plasmid DNAs. Besides, like methotrexate (Fig. 6), cisplatin can further convert the plasmid DNAs into linear DNAs (Fig. 7f-h). The results visually provide novel insights into the dynamic actions of cisplatin. The rapid development of AFM in the past decade has contributed much to the cell and molecular biology [43-45]. AFM imaging especially time-lapse imaging can not only provide "visual evidence" for previously speculated molecular behaviors, but also reveal more detailed behaviors of the individual molecules [12], facilitating us to investigate the relationship between molecular structures and functions.

Fig. 8 shows the changes in geometry of plasmid DNAs after treatment by methotrexate and cisplatin respectively according to the method in Fig. 4. Fig. 8a represents the dynamic changes of geometric features of plasmid DNAs for different concentrations $\left(1,5 \mathrm{ng} \mu \mathrm{L}^{-1}\right)$ and incubation times (30 min, $60 \mathrm{~min}$ ) of methotrexate. We can see that as the increase of drug concentration and incubation time, both of the length and width of plasmid DNAs decrease gradually. Fig. $8 \mathrm{~b}$ is the dynamic changes of geometric features of the plasmid DNAs for incubation times $(30,60$ min) of $5 \mathrm{ng} \mu \mathrm{L}^{-1}$ cisplatin. We can also see that the geometric features (length and width) of the plasmid DNAs decrease as the increase of incubation time. The decrease of the geometric size indicates the shrinkage of plasmid DNAs. The statistical data in Fig. 8 show that the geometric features of plasmid DNAs presented in this work are sensitive to the external drug stimulations and the changes of geometric features are consistent with the morphology of single plasmid DNAs (as the curling and disruption phenomenon observed in Figs 4-7), showing that the geometric features (length, width) extracted from the AFM images may serve as a potential effective and label-free biomarker for indicating the states of plasmid DNAs. By utilizing the geometric parameters, we may further develop quantitative methods to characterize the actions of drugs on DNAs, which may be useful for drug evaluation at the single-molecule level.

\section{CONCLUSION}

In this work, AFM was used to visually and quantitatively investigate the interactions between chemotherapy drugs (methotrexate and cisplatin) and plasmid DNAs at the single-molecule level. By AFM imaging, single plasmid DNAs were clearly discerned on APTES-coated mica and drug-induced changes in morphology and geometry of plasmid DNAs were dynamically revealed. Both drugs resulted in increased local curling of the plasmid DNAs and even disruption of plasmid DNAs into linear DNAs. The geometric features (length, width) of plasmid DNAs extracted from the AFM images decreased significantly after the treatment with methotrexate and cisplatin as the increase of drug concentrations and incubation times. These experimental results deepen our understanding of the actions of methotrexate and cisplatin. Future studies will focus on applying the established procedure to investigate the interactions between plasmid DNAs and more drugs. These studies will be particularly useful for us to quantitatively understand the actions of DNA-related drugs at nanoscale. 
Received 31 October 2016; accepted 19 December 2016; published online 13 January 2017

1 Jemal A, Bray F, Center MM, et al. Global cancer statistics. CA Cancer J Clin, 2011, 61: 69-90

2 DeVita Jr. VT, Rosenberg SA. Two hundred years of cancer research. N Engl J Med, 2012, 366: 2207-2214

3 Nars MS, Kaneno R. Immunomodulatory effects of low dose chemotherapy and perspectives of its combination with immunotherapy. Int J Cancer, 2013, 132: 2471-2478

4 Nitiss JL. Targeting DNA topoisomerase II in cancer chemotherapy. Nat Rev Cancer, 2009, 9: 338-350

5 Dupres V, Alsteens D, Andre G, et al. Fishing single molecules on live cells. Nano Today, 2009, 4: 262-268

6 Lyubchenko YL, Shlyakhtenko LS, Ando T. Imaging of nucleic acids with atomic force microscopy. Methods, 2011, 54: 274-283

7 Ido S, Kimiya H, Kobayashi K, et al. Immunoactive two-dimensional self-assembly of monoclonal antibodies in aqueous solution revealed by atomic force microscopy. Nat Mater, 2014, 13: 264-270

8 Chiaruttini N, Redondo-Morata L, Colom A, et al. Relaxation of loaded ESCRT-III spiral springs drives membrane deformation. Cell, 2015, 163: 866-879

9 Alonso-Sarduy L, Longo G, Dietler G, et al. Time-lapse AFM imaging of DNA conformational changes induced by daunorubicin. Nano Lett, 2013, 13: 5679-5684

10 Krautbauer R, Clausen-Schaumann H, Gaub HE. Cisplatin changes the mechanics of single DNA molecules. Angew Chem Int Ed, 2000, 39: 3912-3915

11 Liu N, Bu T, Song Y, et al. The nature of the force-induced conformation transition of dsDNA studied by using single molecule force spectroscopy. Langmuir, 2010, 26: 9491-9496

12 Kodera N, Yamamoto D, Ishikawa R, et al. Video imaging of walking myosin $\mathrm{V}$ by high-speed atomic force microscopy. Nature, 2010, 468: 72-76

13 Kalle W, Strappe P. Atomic force microscopy on chromosomes, chromatin and DNA: a review. Micron, 2012, 43: 1224-1231

14 Pyne A, Thompson R, Leung C, et al. Single-molecule reconstruction of oligonucleotide secondary structure by atomic force microscopy. Small, 2014, 10: 3257-3261

15 Li M, Liu L, Xi N, et al. Progress in measuring biophysical properties of membrane proteins with AFM single-molecule force spectroscopy. Chin Sci Bull, 2014, 59: 2717-2725

16 Li M, Xiao X, Liu L, et al. Rapid recognition and functional analysis of membrane proteins on human cancer cells using atomic force microscopy. J Immunological Methods, 2016, 436: 41-49

17 Wiggins PA, van der Heijden T, Moreno-Herrero F, et al. High flexibility of DNA on short length scales probed by atomic force microscopy. Nat Nanotech, 2006, 1: 137-141

18 Podesta A, Imperadori L, Colnaghi W, et al. Atomic force microscopy study of DNA deposited on poly l-ornithine-coated mica. J Microsc, 2004, 215: 236-240

19 Hou XM, Zhang XH, Wei KJ, et al. Cisplatin induces loop structures and condensation of single DNA molecules. Nucleic Acids Res, 2009, 37: 1400-1410

20 Liu Z, Tan S, Zu Y, et al. The interactions of cisplatin and DNA studied by atomic force microscopy. Micron, 2010, 41: 833-839

21 Nuttall P, Lee K, Ciccarella P, et al. Single-molecule studies of unlabeled full-length $\mathrm{p} 53$ protein binding to DNA. J Phys Chem B, 2016, 120: 2106-2114

22 Cassina V, Manghi M, Salerno D, et al. Effects of cytosine methylation on DNA morphology: an atomic force microscopy study. BBA-Gen Subjects, 2016, 1860: 1-7
23 Cassina V, Seruggia D, Beretta GL, et al. Atomic force microscopy study of DNA conformation in the presence of drugs. Eur Biophys J, 2011, 40: 59-68

24 Cesconetto EC, Junior FSA, Crisafuli FAP, et al. DNA interaction with Actinomycin D: mechanical measurements reveal the details of the binding data. Phys Chem Chem Phys, 2013, 15: 11070-11077

25 Rafique B, Khalid AM, Akhtar K, et al. Interaction of anticancer drug methotrexate with DNA analyzed by electrochemical and spectroscopic methods. Biosens Bioelectron, 2013, 44: 21-26

26 Heng JB, Aksimentiev A, Ho C, et al. The electromechanics of DNA in a synthetic nanopore. Biophys J, 2006, 90: 1098-1106

27 Liu Z, Li Z, Zhou H, et al. Imaging DNA molecules on mica surface by atomic force microscopy in air and in liquid. Microsc Res Tech, 2005, 66: 179-185

28 Sun L, Zhao D, Zhang Y, et al. DNA adsorption and desorption on mica surface studied by atomic force microscopy. Appl Surface Sci, 2011, 257: 6560-6567

29 Miyagi A, Ando T, Lyubchenko YL. Dynamics of nucleosomes assessed with time-lapse high-speed atomic force microscopy. Biochemistry, 2011, 50: 7901-7908

30 Ficarra E, Masotti D, Macii E, et al. Automatic intrinsic DNA curvature computation from AFM images. IEEE Trans Biomed Eng, 2005, 52: 2074-2086

31 Thomson NH, Santos S, Mitchenall LA, et al. DNA G-segment bending is not the sole determinant of topology simplification by type II DNA topoisomerases. Sci Rep, 2014, 4: 6158

32 Jo K, Dhingra DM, Odijk T, et al. A single-molecule barcoding system using nanoslits for DNA analysis. Proc Natl Acad Sci USA, 2007, 104: 2673-2678

33 Brinkers S, Dietrich HRC, de Groote FH, et al. The persistence length of double stranded DNA determined using dark field tethered particle motion. J Chem Phys, 2009, 130: 215105-215105

34 Brunet A, Tardin C, Salomé L, et al. Dependence of DNA persistence length on ionic strength of solutions with monovalent and divalent salts: a joint theory-experiment study. Macromolecules, 2015, 48: 3641-3652

35 Geggier S, Kotlyar A, Vologodskii A. Temperature dependence of DNA persistence length. Nucleic Acids Res, 2011, 39: 1419-1426

36 Canetta E, Riches A, Borger E, et al. Discrimination of bladder cancer cells from normal urothelial cells with high specificity and sensitivity: combined application of atomic force microscopy and modulated Raman spectroscopy. Acta Biomater, 2014, 10: 2043-2055

37 Linko V, Ora A, Kostiainen MA. DNA nanostructures as smart drug-delivery vehicles and molecular devices. Trends Biotech, 2015, 33: 586-594

38 Wu N, Willner I. pH-stimulated reconfiguration and structural isomerization of origami dimer and trimer systems. Nano Lett, 2016, 16: $6650-6655$

39 van Loenhout MTJ, de Grunt MV, Dekker C. Dynamics of DNA supercoils. Science, 2012, 338: 94-97

40 Oberstrass FC, Fernandes LE, Bryant Z. Torque measurements reveal sequence-specific cooperative transitions in supercoiled DNA. Proc Natl Acad Sci USA, 2012, 109: 6106-6111

41 Pontinha ADR, Jorge SMA, Chiorcea Paquim AM, et al. In situ evaluation of anticancer drug methotrexate-DNA interaction using a DNA-electrochemical biosensor and AFM characterization. Phys Chem Chem Phys, 2011, 13: 5227-5234

42 Dasari S, Bernard Tchounwou P. Cisplatin in cancer therapy: molecular mechanisms of action. Eur J Pharmacology, 2014, 740: $364-378$ 
43 Li M, Liu LQ, Xi N, et al. Effects of temperature and cellular interactions on the mechanics and morphology of human cancer cells investigated by atomic force microscopy. Sci China Life Sci, 2015, 58: 889-901

44 Li M, Xiao X, Liu L, et al. Nanoscale quantifying the effects of targeted drug on chemotherapy in lymphoma treatment using atomic force microscopy. IEEE Trans Biomed Eng, 2016, 63: 2187-2199

45 Ando T, Uchihashi T, Scheuring S. Filming biomolecular processes by high-speed atomic force microscopy. Chem Rev, 2014, 114: 3120-3188

Acknowledgments This work was supported by the National Natu- ral Science Foundation of China $(61503372,61522312,61327014$ and 61433017), the Youth Innovation Promotion Association CAS, and the CAS FEA International Partnership Program for Creative Research Teams.

Author contributions $\mathrm{Li}$ M and Liu L designed the project. Li M performed the experiments and wrote the paper with support from Xi N and Wang Y. Xiao X was involved in data analysis. All authors contributed to the general discussion.

Conflict of interest The authors declare that there is no conflict of interests.

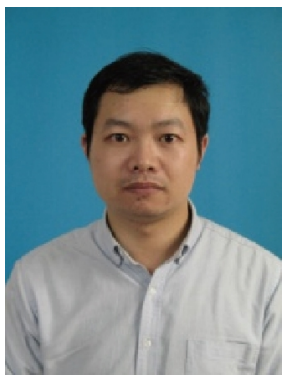

Mi Li is currently an associate professor at the State Key Laboratory of Robotics, Shenyang Institute of Automation, Chinese Academy of Sciences, Shenyang, China. His research interests include AFM-based imaging and mechanical analysis of biological systems.
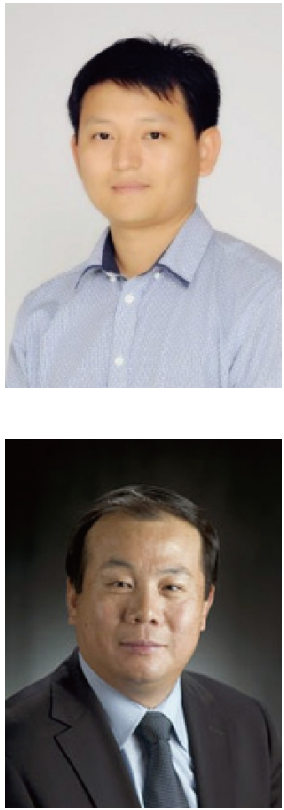

Lianqing Liu is currently a professor at the State Key Laboratory of Robotics, Shenyang Institute of Automation, Chinese Academy of Sciences, Shenyang, China. His research interests include micro/nano system, nanodevice fabrication, nanobiotechnology and biosensors.
Ning Xi is currently a John D. Ryder Professor of Electrical and Computer Engineering at Michigan State University, East Lansing and a professor of Shenyang Institute of Automation, Chinese Academy of Sciences, Shenyang, China. His research interests include robotics, manufacturing automation, nanosensors, micro/nano manufacturing, and intelligent control and systems.

\section{基于AFM的化疗药物与质粒DNA间动态相互作用观测}

李密 ${ }^{1}$, 刘连庆 ${ }^{1 *}$, 肖秀斌 ${ }^{2}$, 席宁 ${ }^{1,3^{*}}$, 王越超 ${ }^{1}$

摘要 原子力显微镜(atomic force microscopy, AFM)的出现为单根DNA分子形貌成像提供了新的技术手段. DNA是许多化疗药物的作用靶点. 尽管研究人员利用AFM对化疗药物与DNA之间的相互作用进行了大量研究, 但对于化疗药物与质粒DNA间动态相互作用过程的认知还很 缺乏. 本文利用AFM研究了纳米尺度下质粒DNA与两种商用化疗药物(甲氨蝶呤, 顺铂)之间的相互作用. 质粒DNA吸附在硅烷化的云母表 面. AFM成像结果清晰地揭示出化疗药物刺激下单根质粒DNA形貌的动态变化. 从AFM图像中提取出质粒DNA的几何特征, 统计结果表明 化疗药物刺激后质粒DNA的几何特征发生了显著变化. 本研究为单分子尺度下研究化疗药物与质粒DNA之间的相互作用提供了新的思路. 